\title{
Bi-level Optimization of Blended Composite Panels
}

\author{
Dianzi Liu* \\ School of Mechanical Engineering, University of Leeds, LS2 9JT, UK \\ Vassili V. Toropov ${ }^{\dagger}$ \\ School of Mechanical Engineering and School of Civil Engineering, University of Leeds, LS2 9JT, UK \\ and \\ Osvaldo M. Querin ${ }^{\ddagger}$ and David C. Barton ${ }^{\S}$ \\ School of Mechanical Engineering, University of Leeds, LS2 9JT, UK
}

\begin{abstract}
Two approaches are examined for finding the best stacking sequence of laminated composite wing structures with blending and manufacturing constraints: smeared stiffnessbased method and lamination parameter-based method. In the first method, the material volume is the objective function at the global level and the stack shuffling to satisfy blending and manufacturing constraints is performed at the local level. The other method introduced in this paper is to use lamination parameters and numbers of plies of the pre-defined angles $(0,90,45$ and -45 degrees) as design variables with buckling, strength and ply percentage constraints while minimizing the material volume in the top level optimization run. Given lamination parameters from the top level optimization as targets for the local level, optimal stacking sequence is determined to satisfy the global blending requirements. On a benchmark problem of an 18-panel wing box, the results from these two approaches are compared to published results to demonstrate their potential.
\end{abstract}

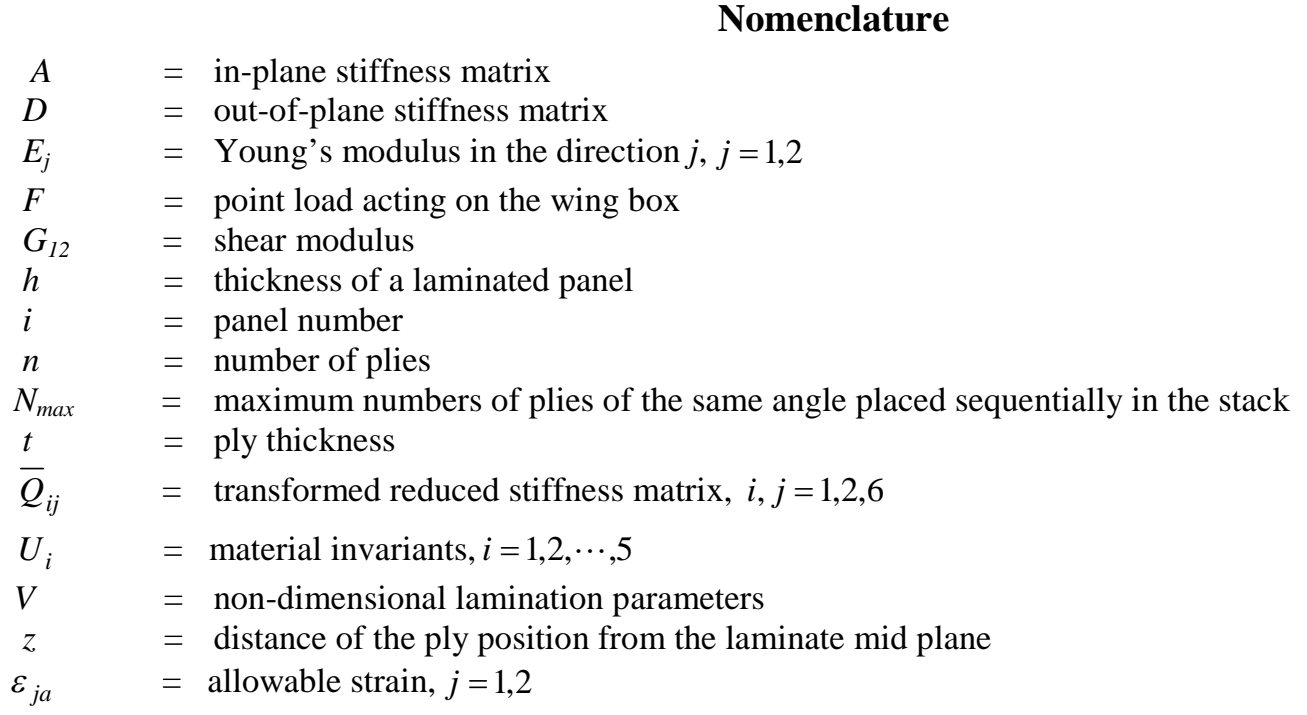

\footnotetext{
* PhD student, School of Mechanical Engineering, University of Leeds, LS2 9JT, UK, AIAA Student Member. ${ }^{\dagger}$ Professor of Aerospace and Structural Engineering, School of Civil Engineering and School of Mechanical Engineering, University of Leeds, LS2 9JT, UK, AIAA Associate Fellow.

* Senior Lecturer, School of Mechanical Engineering, University of Leeds, LS2 9JT, UK, AIAA Senior Member.

${ }^{\S}$ Professor of Solid Mechanics, School of Mechanical Engineering, University of Leeds, LS2 9JT, UK.
} 


$\begin{array}{ll}\gamma_{12 a} & =\text { allowable shear strain } \\ \theta & =\text { ply angle } \\ \rho & =\text { material density } \\ v & =\text { Poisson's ratio }\end{array}$

\section{Introduction}

$\mathrm{T}$ he material volume and the stacking sequence of plies in a composite aircraft structure are of vital importance for achieving the material's required mechanical characteristics such as in-plane, flexural and bucking behaviour ${ }^{1-4}$. Due to the industrial requirements and practical manufacturing considerations, symmetric and balanced laminates with ply orientations of $0,90,45$ and -45 degrees are investigated in this work.

Liu et al. ${ }^{5,6}$ presented a bi-level (global and local) strategy for optimization of a composite wing box structure. At the global level, continuous optimization of thicknesses of $0,90,45$ and -45 degree plies was performed to minimize the weight of a wing box subject to strain and buckling constraints. For a given the number of plies of each orientation and in-plane loads, a permutation genetic algorithm (GA) was used at the local level to optimize the stacking sequence in order to maximize the buckling load. The optimum buckling load, which was treated as a function of the loading and the numbers of plies of $0,90,45$ and -45 degree orientation, was evaluated by a cubic polynomial response surface approximation.

The use of lamination parameters is another approach to represent the in-plane and flexural stiffness in the optimization of laminated composites. It was first used by Tsai et $\mathrm{l}^{7}$ and later applied to the buckling optimization of orthotropic laminated plates by Fukunaga and Hirano ${ }^{8}$. Miki ${ }^{9}$ and Fukunaga ${ }^{10}$ used lamination parameters for tailoring mechanical properties of laminated composites. In a laminated composite optimization problems, lamination parameters can be used as design variables instead of layer thicknesses and ply angles in order to avoid falling into local optima. Diaconu et al. ${ }^{11}$ used a variational approach to determine feasible regions in the space of lamination parameters as constraints in the optimization problem.

Herencia and Weaver ${ }^{12}$ applied a mathematical programming technique and a GA to optimize anisotropic laminated composite panels with T-stiffeners. In the first step, weight optimization based on mathematical programming was performed where the skin and a stiffener were parameterized using lamination parameters, subject to the constraints on buckling, strength as well as practical design rules. A composite layup of a panel was determined using a GA in the second level by meeting the target values of lamination parameters coming from the top level. Herencia et al. ${ }^{13}$ used the same approach for optimization of laminated composite panels with T-stiffeners, but with a different objective function at the second level. Instead of minimizing the squared distance between the target lamination parameters from the first step and the actual lamination parameters, the maximum value of the linearised design constraints was taken as the objective function. The authors' conclusion was that in the determination of the stacking sequence the minimum squared distance might not be the best objective.

Ply compatibility (also referred to as blending) between adjacent panels is a very important consideration in the design of composite structures, it has been considered by Liu and Haftka ${ }^{14}$, Liu et al. ${ }^{5}$, Soremekun et al. ${ }^{15}$ and Seresta et $a l .{ }^{16}$. Liu and Haftka ${ }^{14}$ defined the composition continuity and the stacking sequence continuity measures that were used in an optimization process, also by Toropov et al. ${ }^{17}$ Soremekun et al. ${ }^{15}$ and Seresta $e t a l .{ }^{16}$ developed two blending methods, inward and outward blending, to improve the ply continuity between adjacent panels using a guide based GA. Recently, Liu and $\operatorname{Krog}^{18}$ developed a new approach to identifying a laminate stacking sequence in individual wing panels satisfying inter-panel continuity constraints. In this method, a conventional stacking sequence identification problem was transformed into a problem of shuffling of a set of global ply layout cards. A permutation GA was applied to find an optimal card sequence, which uses the ply angle percentages and the chordwise and spanwise laminate thickness distributions as input data. The authors' conclusion was that it allowed to considerably reduce the design space and hence the solution time.

In this paper, two optimization approaches are used for the optimization of stacking sequence of laminated composite structures: a smeared stiffness-based method and a lamination parameter-based method. In the smeared stiffness-based method, a gradient-based optimization is used to optimize the total mass of the structure at the top level subject to the buckling and strength constraints. A blending scheme and a ply shuffling code based on the layup design rules are applied. In the lamination parameter-based method, the total number of plies and the lamination parameters related to the bending stiffness matrix are treated as the design variables in the top level optimization problem. Buckling and strength constraints are applied at this level and the total mass is the objective function. Next, a permutation GA is used to shuffle the layers to minimize the difference between the values of computed lamination parameters for a current stack and the ones coming from the top level. This is embedded into a blending procedure applied at this level to achieve the global ply continuity. 


\section{Optimization Strategies}

In order to minimize the total weights of all panels, the numbers of plies of each fibre orientation $\left(0^{\circ}, 90^{\circ}, 45^{\circ},-45^{\circ}\right)$ in the panels are defined as design variables in the optimization problem subject to the buckling constraints and constraints on the principal strain values. Industrial requirements and practical manufacturing considerations led to the assumption that only symmetric and balanced laminates with ply orientations $0^{\circ}, 90^{\circ}, 45^{\circ}$ and $-45^{\circ}$ need to be investigated. Therefore, only half the number of plies of each orientation is given in all numerical results presented in this paper. Also, as the number of plies of $45^{\circ}$ angle $\left(n_{45}\right)$ is always equal to the number of plies of $-45^{\circ}$ degree angle $\left(n_{-45}\right)$ for balanced laminates, the number of pairs of $\pm 45^{\circ}$ plies is presented as $n_{ \pm 45}$. At the local level, maximization of ply compatibility will be achieved by the optimization of the ply stacking sequence whereas the overall laminate thickness remains constant as it is fixed after the top level optimization.

\section{A. Smeared Stiffness-Based Method}

Smeared stiffness-based method is an approach that aims to neutralize the stacking sequence effects on the buckling performance by considering homogeneous sections with quasi-isotropic layups. This method is used to calculate the matrices $\mathbf{A}$ and $\mathbf{D}$ of laminates without determining a stacking sequence. Hence no pre-defined stacking sequence of plies is needed in the top level weight optimization. According to the classical laminate theory (CLT) ${ }^{19}$, the matrices $\mathbf{A}$ and $\mathbf{D}$ can be formulated as:

$$
\mathbf{A}=\left[\begin{array}{lll}
A_{11} & A_{12} & A_{16} \\
& A_{22} & A_{26} \\
\text { sym } & & A_{66}
\end{array}\right], \quad \mathbf{D}=\left[\begin{array}{lll}
D_{11} & D_{12} & D_{16} \\
& D_{22} & D_{26} \\
s y m & & D_{66}
\end{array}\right]
$$

where

$$
\begin{aligned}
& A_{i j}=\int_{-h / 2}^{h / 2} \bar{Q}_{i j} d z, \quad i=j=1,2,6, \\
& D_{i j}=\int_{-h / 2}^{h / 2} \bar{Q}_{i j} z^{2} d z, \quad i=j=1,2,6 .
\end{aligned}
$$

The membrane stiffness matrix $\mathbf{A}$ can be rewritten as:

$$
\mathbf{A}=h\left(\sum_{k=1}^{N}\left(\bar{Q}_{i j}\right)_{k} / N\right), \quad i=j=1,2,6 .
$$

With the assumption of material homogeneity above, the bending stiffness matrix $\mathbf{D}$ can be rewritten as:

$$
\mathbf{D}=\bar{Q}_{i j} \int_{-h / 2}^{h / 2} z^{2} d z=\left(\sum_{k=1}^{N}\left(\bar{Q}_{i j}\right)_{k} / N\right) \frac{h^{3}}{12}, \quad i=j=1,2,6
$$

where $N$ is the total number of plies and $h$ is the total thickness of the laminate. Therefore, for a homogeneous material the relationship between $\mathbf{A}$ and $\mathbf{D}$ can be formulated as:

$$
\mathbf{D}=\mathbf{A} h^{2} / 12 .
$$

The application of this approach to the concept optimization of composite structures was demonstrated by Zhou ${ }^{20}$ and implemented in Altair's OptiStruct structural optimization and FE simulation software ${ }^{21}$. In this approach, a bilevel optimization process is used. At the top level, the mass of all plies is the objective function and the design variables are the numbers of plies of each orientation. Both buckling and strength constraints are considered. In this work, Ansys ${ }^{22} \mathrm{FE}$ software is used for calculating strains and the buckling load. As it also incorporates a gradientbased optimization method, it is used for the top level optimization. At the local (bottom) level, the Altair's HyperShuffle ${ }^{20,23}$ software is used for arranging a given number of plies of each orientation (obtained in the top level 
optimization) into a stacking sequence that satisfies the composite design rules. Ply shuffling in the stack is performed in such a way that the stack composition remains as uniform as possible while satisfying the composite design rules. Ply shuffling does not alter the strain values and also the buckling load of the shuffled stack remains quite similar to that of the homogeneous composite material handled in the top level optimization, although this cannot be always guaranteed. The advantage of this method is that it avoids a stack optimization at the local (bottom) level by performing a quicker post-processing function of ply shuffling. The fact that ply shuffling can lead to a (slight) violation of the buckling constraint, particularly when shuffling is performed many times in the blending procedure as described below, can be considered a disadvantage.

\section{B. Lamination Parameter-Based Method}

Lamination parameters were first introduced by Tsai et $a l^{7}{ }^{7}$. It is known that the stiffness matrices $\mathbf{A}$ and $\mathbf{D}$ are governed by 12 lamination parameters and five material parameters. For orthotropic symmetric and balanced laminates, the number of independent lamination parameters can be reduced to eight. The elements of the membrane stiffness matrix $\mathbf{A}$ and the bending stiffness matrix $\mathbf{D}$ can be expressed as:

$$
\begin{aligned}
& {\left[\begin{array}{l}
A_{11} \\
A_{22} \\
A_{12} \\
A_{16} \\
A_{26} \\
A_{66}
\end{array}\right]=h\left[\begin{array}{ccccc}
1 & \xi_{1}^{A} & \xi_{2}^{A} & 0 & 0 \\
1 & -\xi_{1}^{A} & \xi_{2}^{A} & 0 & 0 \\
0 & 0 & -\xi_{2}^{A} & 1 & 0 \\
0 & 0 & -\xi_{1}^{A} & 0 & 1 \\
0 & \xi_{3}^{A} / 2 & \xi_{4}^{A} & 0 & 0 \\
0 & \xi_{3}^{A} / 2 & -\xi_{4}^{A} & 0 & 0
\end{array}\right]\left[\begin{array}{c}
U_{1} \\
U_{2} \\
U_{3} \\
U_{4} \\
U_{5}
\end{array}\right],} \\
& {\left[\begin{array}{l}
D_{11} \\
D_{22} \\
D_{12} \\
D_{16} \\
D_{26} \\
D_{66}
\end{array}\right]=\left(\frac{h^{3}}{12}\right)\left[\begin{array}{ccccc}
1 & \xi_{1}^{D} & \xi_{2}^{D} & 0 & 0 \\
1 & -\xi_{1}^{D} & \xi_{2}^{D} & 0 & 0 \\
0 & 0 & -\xi_{2}^{D} & 1 & 0 \\
0 & 0 & -\xi_{1}^{D} & 0 & 1 \\
0 & \xi_{3}^{D} / 2 & \xi_{4}^{D} & 0 & 0 \\
0 & \xi_{3}^{D} / 2 & -\xi_{4}^{D} & 0 & 0
\end{array}\right]\left[\begin{array}{l}
U_{1} \\
U_{2} \\
U_{3} \\
U_{4} \\
U_{5}
\end{array}\right] .}
\end{aligned}
$$

This suggests that the use of lamination parameters as design variables in the composite optimization can be very beneficial. It is known ${ }^{1,11}$ that the relationship between the out-of-plane lamination parameters can be expressed as:

$$
2\left(1+\xi_{2}^{D}\right)\left(\xi_{3}^{D}\right)^{2}-4 \xi_{1}^{D} \xi_{3}^{D} \xi_{4}^{D}+\left(\xi_{4}^{D}\right)^{2} \leq\left(\xi_{2}^{D}-2\left(\xi_{1}^{D}\right)^{2}+1\right)\left(1-\xi_{2}^{D}\right)
$$

For the majority of aeronautical structures symmetric and balanced laminates with ply orientations of $0,90,45$ and 45 degrees are used. Thus, $\xi_{4}^{D}=0$ and the relationship (6) can be rewritten as:

$$
\left(\xi_{3}^{D}\right)^{2} \leq \frac{\left(\xi_{2}^{D}-2\left(\xi_{1}^{D}\right)^{2}+1\right)\left(1-\xi_{2}^{D}\right)}{2\left(1+\xi_{2}^{D}\right)}
$$

This corresponds to a constraint defining the feasible region for lamination parameters in the optimization of alaminated composite. Here, the non-dimensional lamination parameters are defined as: 


$$
\begin{aligned}
& V_{[0,1,2,3,4], i}^{D}=1+\left(\frac{2}{h_{i}}\right)^{3} \int_{-h_{i} / 2}^{h_{i} / 2}[1, \cos 2 \theta, \sin 2 \theta, \cos 4 \theta, \sin 4 \theta] z^{2} d z \\
& V_{0, i}^{A}=\left(\frac{1}{h_{i}}\right)_{-h_{i} / 2}^{h_{i} / 2} 1 d z=2\left(n_{0}^{i}+n_{90}^{i}+2 n_{45}^{i}\right)=1 \\
& V_{1, i}^{A}=\left(\frac{1}{h_{i}}\right)_{-h_{i} / 2}^{h_{i} / 2} \cos 2 \theta d z=2\left(n_{0}^{i}-n_{90}^{i}\right)=\frac{n_{0}^{i}-n_{90}^{i}}{n_{0}^{i}+n_{90}^{i}+2 n_{45}^{i}} \\
& V_{2, i}^{A}=\left(\frac{1}{h_{i}}\right)_{-h_{i} / 2}^{h_{i} / 2} \sin 2 \theta d z=0 \\
& V_{3, i}^{A}=\left(\frac{1}{h_{i}}\right)_{-h_{i} / 2}^{h_{i} / 2} \cos 4 \theta d z=2\left(n_{0}^{i}+n_{90}^{i}-2 n_{45}^{i}\right)=\frac{n_{0}^{i}+n_{90}^{i}-2 n_{45}^{i}}{n_{0}^{i}+n_{90}^{i}+2 n_{45}^{i}} \\
& V_{4, i}^{A}=\left(\frac{1}{h_{i}}\right)_{-h_{i} / 2}^{h_{i} / 2} \sin 4 \theta d z=0
\end{aligned}
$$

where $A$ indicates membrane effects,

$D$ indicates bending effects,

$i$ is the panel number,

$n_{0}^{i}$ is half the number of $0^{\circ}$ plies in the total stack of the $i^{\text {th }}$ panel,

$n_{ \pm 45}^{i}$ is half the number of pairs of $\pm 45^{\circ}$ plies in the total stack of the $i^{\text {th }}$ panel,

$n_{90}^{i}$ is half the number of $90^{\circ}$ plies in the total stack of the $i^{\text {th }}$ panel,

$h_{i}$ is the total thickness of the panel $i$,

$\theta$ is the ply angle.

In the formulae above the values of $V_{0, i}^{D}=1+2 / 3, V_{4, i}^{D}=1$ can be immediately evaluated, and the following condition holds: $V_{[1,2,3], i}^{D} \geq 0$.

The vector form of lamination parameters related to the out-of-plane stiffness matrix can be written as:

$$
\mathbf{V}_{i}^{D}=V_{[1,2,3], i}^{D}=1+\left(\frac{2}{h_{i}}\right)^{3} \int_{-h_{i} / 2}^{h_{i} / 2}[\cos 2 \theta, \sin 2 \theta, \cos 4 \theta] z^{2} d z .
$$

In this approach, the lamination parameters related to the out-of-plane stiffness matrix $\mathbf{V}_{i}^{D}$ and the numbers of plies of each orientation $\left(n_{0}^{i}, n_{ \pm 45}^{i}, n_{90}^{i}\right)$ are taken as the design variables in the top level. The material volume is the objective function, and the constraints are imposed on buckling, strength, percentages of the numbers of plies of each orientation as well as the feasibility of lamination parameters. Then, in the local level, a stacking sequence optimization is performed by matching the lamination parameters $\mathbf{V}_{i}^{D}$ that came from the top level optimization with the lamination parameters $\tilde{\mathbf{V}}_{i}^{D}$ computed in the local level optimization subject to satisfaction of the composite design rules and manufacturing requirements. A permutation genetic algorithm (permGA) is used for the local level optimization runs carried out iteratively in order to ensure the ply compatibility of adjacent panels as presented in Section IV. A schematic of the optimization process at this level is shown in Figure 1. The advantage of this approach is that there is no need to check whether the strength or buckling constraints have been violated as long as the lamination parameters obtained after the local level optimization match the given lamination parameter values that came from the top level optimization. In the ply compatibility optimization process it is also required to keep the values of lamination parameters in all the stacks (panels) of the whole structure matching the corresponding values that came from the top level optimization.

\section{Composite Design Rules}

According to aircraft industry manufacturing requirements ${ }^{17,24}$, the laminate layup design rules applied to each panel are as follows:

1) The stack is balanced, i.e. the number of $45^{\circ}$ and $-45^{\circ}$ plies is the same in each of the components. 
2) Due to the damage tolerance requirements, the outer plies for the skin should always contain at least one set of $\pm 45^{\circ}$ plies.

3) The number of plies $\left(N_{\max }\right)$ in any one direction placed sequentially in the stack is limited to four.

4) A $90^{\circ}$ change of angle between two adjacent plies is to be avoided, if possible.

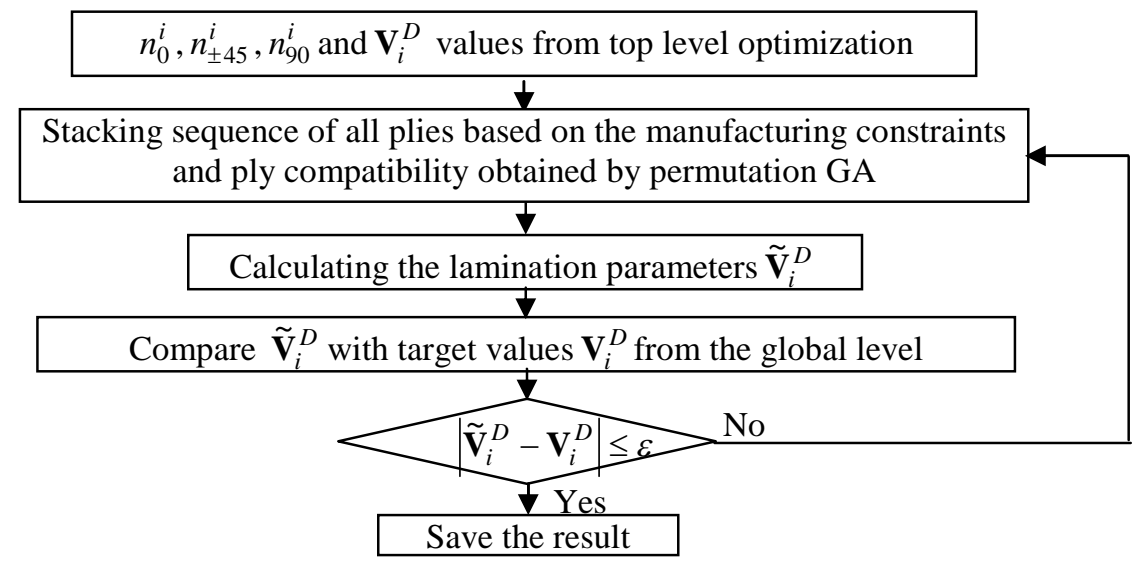

Figure 1. Flow chart of the panel stacking sequence optimization

\section{Shared Layers Blending (SLB)}

In aerospace engineering, a typical wing is a multi-panel tailored composite structure. To improve structural integrity and avoid stress concentration between two adjacent panels, ply blending should be ensured. Although such requirements have been considered by several research groups ${ }^{14-18}$, a problem of optimization of multi-panel aircraft structures with a comprehensive consideration of buckling, strength, manufacturing constraints as well as general composite design rules including ply blending still remains to be addressed to satisfaction of aircraft industry.

In this section the Shared Layers Blending (SLB) process is applied to satisfy the global blending requirement as well as the general layup design rules. Two illustrative examples are given to demonstrate this process.

First, ranking of all panels in terms of the numbers of plies of each angle is performed. Then, for each ply angle, out of all panels the minimum number of plies is selected. This set of three ply numbers defines the first set of shared layers among all panels. The thinnest panel that includes the first shared layers is identified. The first shared layers will be placed outermost in the stacks for all panels. The remaining layers in the thinnest panel are placed after the first shared layers. Next, after this first stage, for the remaining layers of all the panels, except the thinnest panel, the same procedure is applied as at the first stage. This is repeated until the last panel is considered. Finally, for the adjacent panels, the local blending between them is performed for the remaining layers in the adjacent panels. The scheme for the local blending consideration is given in the Example 2. Thus, the stacks for all the panels will become inwardly blended (outer blending), where the outer layers of all the panels are continuous. If the shared layers are placed at the position next to the mid plane instead of the outermost position, the inner blending (outwardly blended composite) will be created. In this paper, the outer blending procedure is adapted due to the damage tolerance requirements resulting in $\pm 45^{\circ}$ plies places on the outside of the stack.

\section{Example 1}

In the first example three panels are linked together with the numbers of plies of each orientation (as coming from the top level optimization) given in Table 1. A flowchart of this approach is shown in Figure 2.

Table 1. Numbers of plies of each orientation for a three-panel laminated structure

\begin{tabular}{|l|l|l|l|}
\hline Panel number & panel 1 & panel 2 & panel 3 \\
\hline $\begin{array}{l}\text { Number of plies } \\
\left(n_{0} / n_{ \pm 45} / n_{90}\right)\end{array}$ & $40 / 17 / 7$ & $35 / 14 / 9$ & $29 / 6 / 12$ \\
\hline
\end{tabular}

Using this approach, the first set of shared layers in this example will be $n_{0} / n_{45} / n_{90}=29 / 6 / 7$ for all three panels and also panel 3 is selected as the first (thinnest) panel. The second shared set will be $n_{0} / n_{45} / n_{90}=6 / 8 / 0$ for the panels 1 and 2 only. Now the remaining layers in the three-panel structure will be: $n_{0} / n_{45} / n_{90}=5 / 3 / 0$ for panel 1 , $n_{0} / n_{45} / n_{90}=0 / 0 / 2$ for panel 2 , and $n_{0} / n_{45} / n_{90}=0 / 0 / 5$ for panel 3 . In this example, because no shared layers are 
available between the panels 1 and 2, the local shared set will be $n_{0} / n_{45} / n_{90}=0 / 0 / 2$ for the remaining layers in the panels 2 and 3. Note that for a case of a panel that is adjacent to several panels, the scheme for consideration of local blending of remaining layers among these panels will be shown in Example 2 below. Thus, the remaining layers in the panel 1 after the SLB procedure will be $n_{0} / n_{45} / n_{90}=5 / 3 / 0$, no remaining layers left in the panel 2 , and $0 / 0 / 3$ in the panel 3. The blending schematic is shown in Figure 3.

\section{Stack Repair}

Two issues arise from the results in Example 1 that need to be addressed in the blending scheme. The first one is that the group of remaining layers in the panel 3 consists only of five 90 degree plies that violates the ply composition rule. The second issue is that the total number of plies in the second set of shared layers truncated between the adjacent panels 2 and 3 can be considered too large (23 plies).

For the first issue, having five 90 degree plies as remaining layers in panel 3 means that too many plies were selected for the first set of shared layers. A slightly larger number of plies including at least one 0 or \pm 45 degree ply has to be included into the set of remaining layers for the panel 3. Therefore, having more than four plies of the same orientation together can be avoided in the panel 3 by reserving some layers from the first set of shared layers. For the second issue, let's assume that the number of truncated plies between the panels 2 and 3 is too large. This means that too many plies are selected as the second set of shared layers in the above blending procedure. Thus, the number of plies used as the second set of shared layers has to be re-adjusted to satisfy the requirement. Generally, for practical stack compositions that satisfy realistic constraints on ply orientation percentages, a solutions for a stack repair can always be found: If a problem happens at a certain stage, the algorithm steps back and plies are removed from the previous set of shared layers in the above blending scheme. This is repeated iteratively until the obtained stack satisfies all the design rules and constraints.

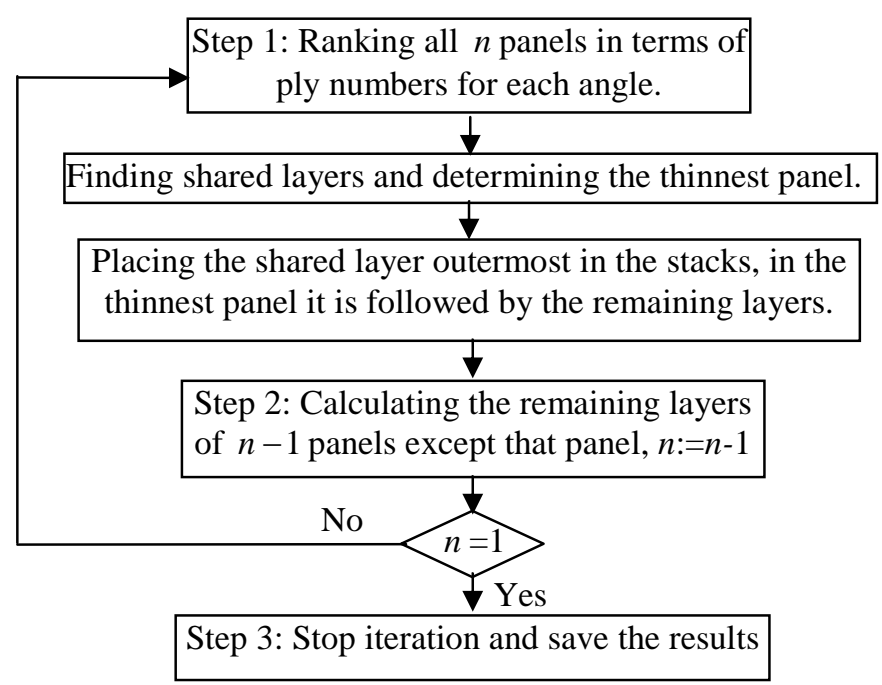

Figure 2. Flowchart for the shared layers blending scheme.

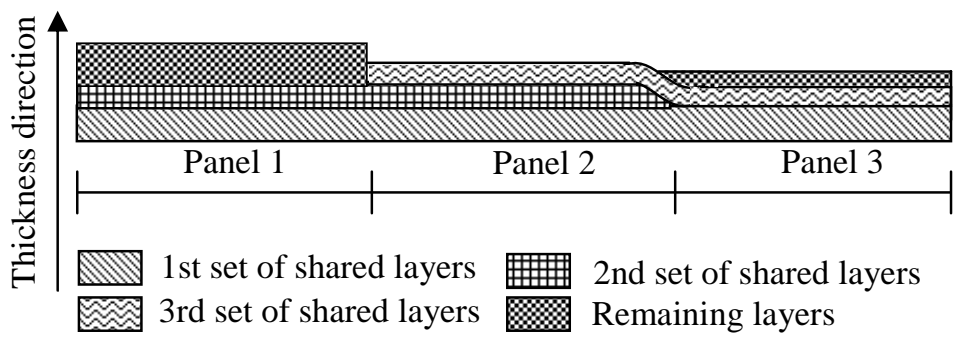

\section{Figure 3. Illustration of shared layers blending concept for the} three-panel linked structure.

\section{Example 2}

The neighbour panel selection scheme for local blending will be explained in this example. A six-panel structure with the numbers of plies of each orientation is shown in Figure 4. In this example, the ranking order of the thicknesse for all six panels is from panel 6 (thinnest) to panel 1 (thickest). Using the blending scheme above, the first set of shared layers in this example will be $n_{0} / n_{45} / n_{90}=13 / 2 / 2$ for all six panels and the panel 6 is selected as the first (thinnest) panel. Next, the second set of shared layer is $n_{0} / n_{45} / n_{90}=1 / 0 / 0$ for all the other five panels (from panel 1 to panel 5). Then, the third set of shared layers is $n_{0} / n_{45} / n_{90}=0 / 3 / 0$ for all the other fours panels (from panel 1 to panel 4). The fourth set of shared layers is $n_{0} / n_{45} / n_{90}=1 / 1 / 1$ for the remaining three panels (from panel 1 to panel 3). The last (fifth) set of shared layers is $n_{0} / n_{45} / n_{90}=0 / 5 / 0$ for panels 1 and 2 . Thus, the remaining layers can be listed as: panel 1: $n_{0} / n_{45} / n_{90}=0 / 1 / 1$; panel $2: n_{0} / n_{45} / n_{90}=1 / 0 / 0$; panel $3: n_{0} / n_{45} / n_{90}=2 / 0 / 2$; panel 4 : 
$n_{0} / n_{45} / n_{90}=0 / 0 / 0$; panel 5: $n_{0} / n_{45} / n_{90}=1 / 0 / 3$ and panel 6: $n_{0} / n_{45} / n_{90}=0 / 0 / 1$. The order of considering the local blending for adjacent panels such as the pair of panels 5 and 6 and the pair of panels 5 and 3 will be determined by the corresponding thickness jump. The latter means the difference of the thicknesses of the two adjacent panels that is known from the top level optimization. Since the thickness jump in the horizontal direction is smaller than that in the vertical direction in this example, the local blending between the panels 5 and 6 will be considered before the blending between the panels 3 and 5. When several adjacent panels follow the same direction, for example, the adjacent panels 1 and 3 and the adjacent panels 3 and 5 follow the vertical direction, the order of local blending will follow the direction which reflects the distributions of the panel thickness: from the thinnest to the thickest. This means that the direction will be from the panel 5 to the panel 1, through the panel 3 . Thus, the local blending between the panels 3 and 5 will be considered before the blending between the panels 1 and 3 .

\begin{tabular}{|c|c|c|c|}
\hline & \multirow{2}{*}{ Panel no. } & \multirow{2}{*}{$\begin{array}{l}\text { Number of plies } \\
\left(n_{0} / n_{ \pm 45} / n_{90}\right)\end{array}$} \\
\hline \multirow{2}{*}{1} & \multirow[t]{2}{*}{2} & & \\
\hline & & 1 & $15 / 12 / 4$ \\
\hline \multirow[t]{2}{*}{3} & \multirow[t]{2}{*}{4} & 2 & $16 / 11 / 3$ \\
\hline & & 3 & $17 / 6 / 5$ \\
\hline \multirow[t]{2}{*}{5} & \multirow[t]{2}{*}{6} & 4 & $14 / 5 / 2$ \\
\hline & & 5 & $15 / 2 / 5$ \\
\hline \multicolumn{2}{|c|}{ (a) } & 6 & $13 / 2 / 3$ \\
\hline
\end{tabular}

(b)

Figure 4. Illustration of local blending concept for six-panel laminated structure, (a) geometry of the structure, (b) numbers of plies of each orientation.

Table 2. Numbers of plies of each orientation and lamination parameters for a three-panel laminated structure

\begin{tabular}{|c|c|c|c|}
\hline & panel 1 & panel 2 & panel 3 \\
\hline $\begin{array}{c}\text { Number of plies } \\
\left(n_{0} / n_{ \pm 45} / n_{90}\right)\end{array}$ & $15 / 4 / 3$ & $12 / 3 / 6$ & $10 / 3 / 5$ \\
\hline$V_{1}^{D}$ & 1.1203 & 1.1400 & 1.2034 \\
\hline$V_{2}^{D}$ & 1.0092 & 1.0056 & 1.0078 \\
\hline$V_{3}^{D}$ & 1.2032 & 1.2932 & 1.0728 \\
\hline
\end{tabular}

\section{Calculation of Lamination Parameters in the Blending Scheme}

In the optimization using the lamination parameter-based method, lamination parameters are calculated at the local level to match the target values from the top level. The lamination parameters are calculated simultaneously with the blending scheme described in the section IV. Once the first set of shared layers is determined by the blending scheme, the stacking sequence for the first set of shared layers will be obtained by a permutation GA to match the lamination parameters from the top level in the thinnest panel. Following that the values of lamination parameters corresponding to the first set of shared layers in each of the remaining panels are calculated. Generally, these values will be different in different panels because, following the outer blending scheme, the distance of the shared layers from the mid-plane varies from panel to panel. Next, the same blending scheme of determining the second set of shared layers is applied. The stacking sequence of the second set of shared layers will be determined to match the difference between the lamination parameters from the top level and the values already calculated for the first set of shared layers in the next thinnest panel (because the first thinnest panel has already been dealt with). This is repeated until the last set of shared layers is considered. Then, lamination parameters contributed from the sets of shared layers are summed up for each panel. Finally, the stacking sequence of remaining layers in each panel will be determined to minimize the difference between the lamination parameters from the top level and the ones summed up in the blending scheme. An example below will demonstrate this procedure.

\section{Example 3}

In this example three panels are sequentially linked with the numbers of plies of each orientation and lamination parameters related to the bending stiffness matrix (as coming from the top level optimization) given in Table 2.

The first set of shared layers in this example will be $n_{0} / n_{45} / n_{90}=10 / 3 / 3$ for all three panels and also panel 3 is selected as the first (thinnest) panel. The stacking sequence of the first set of shared layers will be obtained by the permutation GA to match the lamination parameters from the top level $\left(\mathbf{V}_{i}^{D}=\left[V_{1, i}^{D}, V_{2, i}^{D}, V_{3, i}^{D}\right]\right)$ for panel 3. The values $\tilde{\mathbf{V}}_{i,(j)}^{D}$ will represent the contribution of the lamination parameters calculated for the $j^{\text {th }}$ set of shared layers in the $i^{\text {th }}$ panel. In this example, the lamination parameters calculated for the first set of shared layers in the panel 3 are $\tilde{\mathbf{V}}_{3,(1)}^{D}=[1.1920,1.0068,1.0718]$. At the same time, the contribution of lamination parameters from the stacking sequence of the first set of shared layers to panels 2 and 1 can be calculated as: $\tilde{\mathbf{V}}_{2,(1)}^{D}=[1.1632,1.0038,1.2808]$ and 
$\tilde{\mathbf{V}}_{1,(1)}^{D}=[1.1430,1.0068,1.1878]$, respectively. After the first blending stage, the differences between the lamination parameters from the top level and the values calculated from the first set of shared layers in the panels 2 and 1 are: $\mathbf{V}_{2}^{D}-\tilde{\mathbf{V}}_{2,(1)}^{D}=[-0.0232,0.0018,0.0124]$ and $\mathbf{V}_{1}^{D}-\tilde{\mathbf{V}}_{1,(1)}^{D}=[-0.0227,0.0024,0.0154]$, respectively. Then, the stacking sequence of the second set of shared layers $\left(n_{0} / n_{45} / n_{90}=2 / 0 / 0\right)$ will be determined to target the difference in the panel 2 (although the stacking sequence is trivial in this case). The contributions of lamination parameters calculated from the stacking sequence of the second set of shared layers to panels 2 and 1 are: $\tilde{\mathbf{V}}_{2,(2)}^{D}=[-0.0032,0.0010,0.0114]$ and $\tilde{\mathbf{V}}_{1,(2)}^{D}=[-0.049,0.0012,0.0134]$, respectively. The remaining layers after the global blending scheme are: for the panel 1: $n_{0} / n_{45} / n_{90}=3 / 1 / 0$; for the panel $2: n_{0} / n_{45} / n_{90}=0 / 0 / 3$; for the panel $3: n_{0} / n_{45} / n_{90}=0 / 0 / 2$. Now the local blending will be applied to determine the third set of shared layers between the panels 3 and 2 that is $n_{0} / n_{45} / n_{90}=0 / 0 / 2$. The stacking sequence of the third set of shared layers will be determined to target the difference in the panel 3. The difference between the lamination parameters from the top level and the values calculated from the first set of shared layers is: $\mathbf{V}_{3}^{D}-\tilde{\mathbf{V}}_{3,(1)}^{D}=[0.0114,0.0010,0.0010]$. The contributions of the lamination parameters from the stacking sequence of the third set of shared layers to the panels 2 and 3 can be calculated as: $\tilde{\mathbf{V}}_{3,(3)}^{D}=[0.0010,0.0002,0.0006]$ and $\tilde{\mathbf{V}}_{2,(3)}^{D}=[0.0012,0.0005,0.0008]$, respectively. Finally, the remaining layers after the blending are: for the panel $1: n_{0} / n_{45} / n_{90}=3 / 1 / 0$; for the panel $2: n_{0} / n_{45} / n_{90}=0 / 0 / 1$ and no remaining layers are left for the panel 3. The summation of the lamination parameters for each panel will produce: for the panel 1: $\tilde{\mathbf{V}}_{1,(1)}^{D}+\tilde{\mathbf{V}}_{1,(2)}^{D}=[1.0940,1.0080,1.2012]$; for the panel 2: $\tilde{\mathbf{V}}_{2,(1)}^{D}+\tilde{\mathbf{V}}_{2,(2)}^{D}+\tilde{\mathbf{V}}_{2,(3)}^{D}=[1.1612,1.0053,1.2930]$ and for the panel 3: $\tilde{\mathbf{V}}_{3,(1)}^{D}+\tilde{\mathbf{V}}_{3,(3)}^{D}=[1.1930,1.0070,1.0724]$. For the panel 3, there is no remaining layers left and the calculated lamination parameters are $\tilde{\mathbf{V}}_{3}^{D}=[1.1930,1.0070,1.0724]$. The stacking sequences of the remaining layers in the panels 2 and 1 will be obtained to target the difference between the lamination parameters $\mathbf{V}_{i}^{D}$ from top level and the ones summed up in the blending scheme, respectively.

\section{Permutation GA}

In the lamination parameter-based method, lamination parameters related to the out-of-plane stiffness matrix are obtained from the top level optimization. Given these values, a stacking sequence finding while satisfying the layup rules and the requirements of the blending scheme should be performed. A permutation GA is an ideal tool for such a composite laminate optimization problem. Each string in the coding represents a unique stacking sequence. An example of using the genetic operators with a permutation encoding is given below.

1) Mutation - two numbers are selected and exchanged e.g. $3^{\text {nd }}$ and $5^{\text {th }}$ :

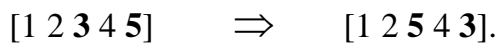

2) Crossover can be done in a variety of ways, such as 'simple crossover', 'cycle crossover', 'inversion' and 'swap adjacent cells'. The 'swap adjacent cells' method, implemented in this work, is illustrated below:

\section{$\left[\begin{array}{lllll}1 & 2 & 3 & 4 & 5\end{array}\right] \quad \Rightarrow \quad\left[\begin{array}{llll}1 & 3 & 2 & 4\end{array}\right]$.}

Also, to reflect the layup rules of composite laminate design and manufacturing requirements, substrings that represent stacks of layers such as $45 / 0 /-45,45 / 90 /-45,45 / 0_{2} /-45$ and $45 / 90_{2} /-45$ are implemented in the permutation GA coding in order to improve the stacking sequence design of composite laminates.

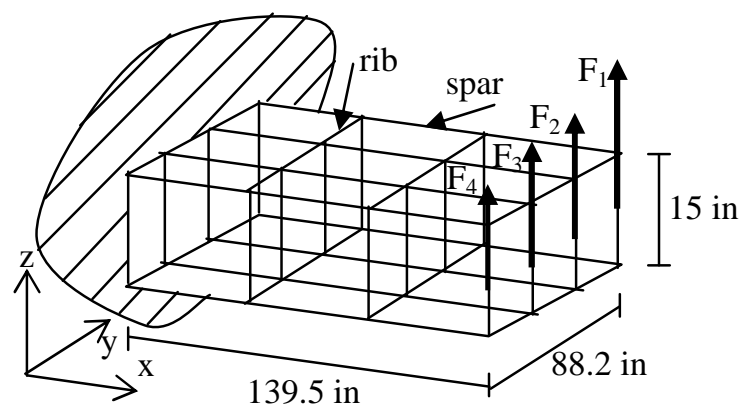

Figure 5. Geometry of the wing box
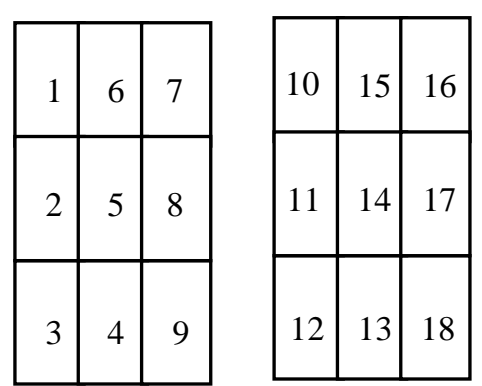

Figure 6. Bottom and top skin configurations 


\section{Wing Box Example}

The wing box model used to illustrate the methods discussed in previous sections is shown in Figures 5 and 6. The material properties are shown in Table 3. Four point loads $F_{1}, F_{2}$, $F_{3}$ and $F_{4}$ with magnitudes $380176.16 \mathrm{~N}$, 187888.44 N, 187888.44 N, and 90009.77 N are applied at the central line of the rib at the free end to avoid the local stress effects on the top skin. These loads induce both upward bending and twisting of the wing box. Due to the aircraft industry manufacturing requirements, 0 or 90 degree plies are required to be inserted into pairs of \pm 45 plies to avoid $90^{\circ}$ change between two adjacent plies. Thus, the bending-twisting coupling terms $D_{16}$ and $D_{26}$ are nonzero from Table 3. Material properties for graphite-epoxy: T300/N5208.

\begin{tabular}{ll}
\hline Material properties & Values \\
\hline Young's Modulus in direction1, $E_{1}$ & $127.56 \mathrm{GPa}$ \\
Young's Modulus in direction2, $E_{2}$ & $13.03 \mathrm{GPa}$ \\
Shear Modulus, $G_{12}$ & $6.41 \mathrm{GPa}$ \\
Poisson's ratio, $v_{12}$ & 0.3 \\
Material density, $\rho$ & $1577.76 \mathrm{~kg} / \mathrm{m}^{3}$ \\
Ply thickness, $t$ & $0.127 \mathrm{~mm}$ \\
Allowable strain in fiber direction $\varepsilon_{1 a}$ & 0.08 \\
Allowable strain in transverse direction $\varepsilon_{2 a}$ & 0.029 \\
Allowable shear strain $\gamma_{12}$ & 0.015 \\
Safety factor & 1.5 \\
\hline
\end{tabular}
the contributions of off-axis layers and the distance of the positive and negative angle plies from the laminate center plane. In this work, the number of 45 degree plies in the top skin and the number of 0 and 90 degree plies in the bottom skin are rounded up to achieve the discrete optimal design.

\section{A. Problem with Two Designable Substructures}

If the layup of all panels in the top skin is the same and all the bottom skin panels are also are the same, the total number of design variables for the wing box is six $\left(n_{0}^{t}, n_{ \pm 45}^{t}, n_{90}^{t}, n_{0}^{b}, n_{ \pm 45}^{b}\right.$ and $n_{90}^{b}$ where superscript $t$ means the top skin and $b$ stands for the bottom skin) in the smeared stiffness-based method. In the lamination parameter-based method six additional non-dimensional design variables $\left(V_{1}^{D}\right)^{t},\left(V_{2}^{D}\right)^{t},\left(V_{3}^{D}\right)^{t},\left(V_{1}^{D}\right)^{b},\left(V_{2}^{D}\right)^{b}$ and $\left(V_{3}^{D}\right)^{b}$ related to the bending stiffness matrix are needed. The results for the objective function and the violation of constraints at the top level are shown in Table 4 for the smeared stiffness-based method and Table 6 for the lamination parameterbased method. Considering the smeared stiffness-based method first, for the continuous optimal design the buckling constraint is active for the panel 16. The top skin is much heavier than the bottom skin due to the buckling constraints. In contrast with the results from Liu et al..$^{5}$, the objective function is reduced to 186 as compared to 208. In the local level optimization, shuffling all the layers from the top level optimization is performed according to the layups rules and the result is shown in Table 5. For the optimal design with the lamination parameter-based method, a lighter structure has been obtained than the one with smeared stiffness-based method. In the local level optimization, given the lamination parameters from the top level, a permutation GA is used to obtain the stacking sequence for the top and bottom skin as presented in Table 7. Buckling constraints are relevant for the top skin and the lamination parameters remain almost same after the local level optimization except for the $V_{2}$ value. The lamination parameters related to the bending stiffness in the bottom skin do not have a significant effect on the buckling of the top skin because the bottom skin is mainly subjected to shear and tensile loads.

Table 4. Continuous and rounded optimal design with 6 variables for smeared stiffness-based method

\begin{tabular}{|c|c|c|c|c|c|c|c|}
\hline & $\begin{array}{l}n_{0} \\
\text { (Cont }\end{array}$ & $\begin{array}{l}n_{45} \\
\text { nuous) }\end{array}$ & $n_{90}$ & $\begin{array}{l}n_{0} \\
\text { (R }\end{array}$ & $\begin{array}{r}n_{45} \\
\text { ded) }\end{array}$ & $n_{90}$ & Active constraints \\
\hline Top skin panels & 40.95 & 10.45 & 16.39 & 41 & 11 & 16 & Panel 16 (buckling) \\
\hline Bottom skin panels & 5.67 & 1.48 & 5.17 & 6 & 1 & 6 & \\
\hline Buckling load factor & 0.990 & & & 1. & & & \\
\hline Total number of plies & 184.1 & & & 18 & & & \\
\hline Total number of plies ${ }^{5}$ & 208.7 & & & 20 & & & \\
\hline
\end{tabular}

Table 5. Stacking sequence of top and bottom skin panels for the rounded design with 6 variables

\begin{tabular}{ll}
\hline Panel no. & \multicolumn{1}{c}{ Stacking sequence } \\
\hline 16 & {$\left[( \pm 45)_{2} /\left(0_{4} / 90 / 45 / 0 /-45\right)_{3} /\left(0_{4} / 90 / 45 / 90 /-45\right)_{5} / 0_{4} / 90 / 45 / 0 /-45 / 90_{2} / 0\right]_{\mathrm{s}}$} \\
7 & {$\left[ \pm 45 / 0_{3} / 90_{3} / 0_{2} / 90_{3} / 0\right]_{\mathrm{s}}$} \\
Buckling load factor & 1.020
\end{tabular}


Table 6 Continuous and rounded optimal design with 12 variables for lamination parameter-based method

\begin{tabular}{|c|c|c|c|c|c|c|c|c|c|}
\hline & $\begin{array}{l}n_{0} \\
\text { (Contir }\end{array}$ & $\begin{array}{c}n_{45} \\
\text { huous) }\end{array}$ & $n_{90}$ & $\begin{array}{l}n_{0} \\
(\mathrm{Ro} \\
\end{array}$ & $\begin{array}{l}n_{45} \\
\text { aded } \\
\end{array}$ & $n_{90}$ & $V_{1}$ & $V_{2}$ & $V_{3}$ \\
\hline Top skin panels & 34.492 & 7.445 & 26.139 & 34 & 8 & 26 & 0.9434 & 1.0065 & 1.2108 \\
\hline Bottom skin panels & 8.163 & 1.480 & 2.181 & 9 & 1 & 3 & 0.8944 & 1.0435 & 0.9710 \\
\hline Buckling load factor & 1.0009 & & & 1.0 & & & & & \\
\hline Total number of plies & 177.65 & & & 180 & & & & & \\
\hline Total number of plies ${ }^{5}$ & 208.76 & & & 208 & & & & & \\
\hline
\end{tabular}

Table 7 Stacking sequence and lamination parameters of the panel at local level with 12 variables

\begin{tabular}{lrrrc}
\hline Panel no. & $V_{1}$ & $V_{2}$ & $V_{3}$ & Buckling load factor \\
\hline 16 & 0.9434 & 1.0080 & 1.2107 & 1.0178 \\
7 & 1.0131 & 1.0190 & 1.1730 & \\
Stacking sequence: & & & & \\
16 & {$\left[( \pm 45)_{2} / 90 / 0 / 45 / 90_{2} /-45 / 90_{2} / 0_{3} / 90 / 0 / 45 / 90 /-45 / 90 / 0 / 45 / 90_{2} /-45 / 0 / 90 / 45 / 90_{2} /-45 / 0_{3} / 90 / 0 / 90 / 45 / 90_{2} /-45 /\right.$} \\
7 & $\begin{array}{l}\left.\text { [ } / 90 / 0_{2} / 90 / 0_{4} / 90 / 0_{4} / 90 / 0 / 90 / 0_{2} / 90_{2} / 0_{2} / 90 / 0 / 90 / 0_{4} / 45 / 0_{2} /-45\right]_{\mathrm{s}} \\
\end{array}$ \\
\end{tabular}

\section{B. Problem with Six Designable Substructures}

If the top and bottom skins are divided into three parts: root, intermediate and tip part, the results are listed in Tables 8, 9, 10 and 11. The weight is reduced considerably as compared to the case of two designable substructures. Results in Tables 8 and 9 correspond to the smeared stiffness-based method. The objective function is reduced to 460 as compared to 464 that is the result of Liu et al. ${ }^{5}$. It should be noted that when the lamination parameter-based method was used shear buckling in the bottom skin occurred (buckling load reduction by 4\%) as shown in Table 11 . This is due to the application of a blending procedure to a part of the structure that has a relatively few plies in which case blending caused a poor match between the target and obtained values of lamination parameters. This can be repaired by adding some layers manually. The second buckling mode corresponds to the top skin, the magnitude of the load factor is close to the value from the top level optimization. This is guaranteed by arriving at a good match with the lamination parameters from top level optimization when a local optimization is performed. Summarising, due to the limited number of plies in the bottom panel, it was difficult to shuffle the plies to match the lamination parameters from the top level while satisfying ply continuity in the bottom skin. For the top skin (that has a much greater number of plies), the lamination parameters are quite close to the ones from the top level optimization and the outer blending with the layup rules requirements did not cause any problems.

Table 8. Continuous and rounded optimal design with 18 variables for smeared stiffness-based method

\begin{tabular}{|c|c|c|c|c|c|c|c|}
\hline & $\begin{array}{l}n_{0} \\
\text { (Cont }\end{array}$ & \multicolumn{2}{|c|}{ (Continuous) } & $\begin{array}{l}n_{0} \\
(\mathrm{R}\end{array}$ & $\begin{array}{c}n_{45} \\
\text { nded) }\end{array}$ & $n_{90}$ & Active constraints \\
\hline \multicolumn{8}{|l|}{ Top skin panels } \\
\hline Panel no. 16 & 30.20 & 12.54 & 24.56 & 30 & 13 & 25 & \\
\hline Panel no. 17 & 18.69 & 20.53 & 12.10 & 19 & 21 & 12 & \\
\hline Panel no.18 & 24.43 & 5.40 & 8.92 & 24 & 6 & 9 & buckling \\
\hline \multicolumn{8}{|l|}{ Bottom skin panels } \\
\hline Panel no.7 & 1.50 & 1.32 & 1.45 & 2 & 1 & 2 & \\
\hline Panel no.8 & 2.38 & 1.01 & 1.32 & 3 & 1 & 2 & \\
\hline Panel no.9 & 7.81 & 3.06 & 3.35 & 8 & 3 & 4 & \\
\hline Buckling load factor & \multicolumn{3}{|l|}{0.9960} & \multicolumn{3}{|c|}{1.0440} & \\
\hline Total number of plies & \multicolumn{3}{|l|}{448.82} & \multicolumn{3}{|c|}{460} & \\
\hline Total number of plies ${ }^{5}$ & \multicolumn{3}{|l|}{465.63} & \multicolumn{3}{|c|}{464} & \\
\hline
\end{tabular}


Table 9 Stacking sequence of top and bottom skin panels for the rounded design with 18 variables

\begin{tabular}{cl}
\hline Panel no. & \multicolumn{1}{c}{ Stacking sequence } \\
\hline 16 & {$\left[( \pm 45)_{2} /\left(0_{4} / 90 / 45 / 0 /-45\right)_{2} /\left(0_{4} / 90 / 45 / 90 /-45\right)_{2} / 90_{2} / 0 /( \pm 45)_{3} /\left(90 /( \pm 45)_{4} /\left(90_{4} / 0_{3}\right)_{3} / 90 / 0_{2}\right]_{\mathrm{s}}\right.$} \\
17 & {$\left[( \pm 45)_{2} /\left(0_{4} / 90 / 45 / 0 /-45\right)_{2} /\left(0_{4} / 90 / 45 / 90 /-45\right)_{2} / 90_{2} / 0 /( \pm 45)_{3} /(90 / \pm 45)_{4} /( \pm 45)_{8}\right]_{\mathrm{s}}$} \\
18 & {$\left[( \pm 45)_{2} /\left(0_{4} / 90 / 45 / 0 /-45\right)_{2} /\left(0_{4} / 90 / 45 / 90 /-45\right)_{2} / 90_{2} / 0_{4} / 90 / 0_{2}\right]_{\mathrm{s}}$} \\
7 & {$\left[ \pm 45 / 90_{2} / 0_{2}\right]_{\mathrm{s}}$} \\
8 & {$\left[ \pm 45 / 90 / 0 / 90 / 0_{2}\right]_{\mathrm{s}}$} \\
9 & {$\left[ \pm 45 / 90 / 0 / 90 / 0_{2} /( \pm 45)_{2} / 0_{4} / 90_{2} / 0\right]_{\mathrm{s}}$}
\end{tabular}

Buckling load factor $\quad 1.019$

Table 10 Continuous and rounded optimal design with 36 variables for lamination parameter-based method

\begin{tabular}{|c|c|c|c|c|c|c|c|c|c|}
\hline & $\begin{array}{l}n_{0} \\
\text { (Cont }\end{array}$ & $\begin{array}{c}\quad n_{45} \\
\text { inuous) }\end{array}$ & $n_{90}$ & $\begin{array}{l}n_{0} \\
\quad(\mathrm{~F}\end{array}$ & $\begin{array}{l}n_{45} \\
\text { und }\end{array}$ & $n_{90}$ & $V_{1}$ & $V_{2}$ & $V_{3}$ \\
\hline \multicolumn{10}{|l|}{ Top skin panels } \\
\hline Panel no.16 & 30.20 & 12.54 & 24.56 & 28 & 16 & 22 & 1.1268 & 1.0102 & 1.2132 \\
\hline Panel no. 17 & 18.69 & 20.53 & 12.10 & 26 & 13 & 19 & 1.1610 & 1.0086 & 1.3022 \\
\hline Panel no.18 & 24.43 & 5.40 & 8.92 & 22 & 6 & 14 & 1.2398 & 1.0098 & 1.0982 \\
\hline \multicolumn{10}{|l|}{ Bottom skin panels } \\
\hline Panel no.7 & 4.39 & 1.30 & 1.28 & 5 & 1 & 1 & 1.3715 & 1.0579 & 0.7382 \\
\hline Panel no.8 & 3.92 & 1.20 & 2.06 & 4 & 1 & 2 & 1.1144 & 1.0576 & 0.7906 \\
\hline Panel no.9 & 7.48 & 1.72 & 2.68 & 8 & 2 & 3 & 0.8432 & 1.0485 & 0.9308 \\
\hline Buckling load factor & \multicolumn{3}{|l|}{1.0039} & \multicolumn{6}{|c|}{1.0349} \\
\hline Total number of plies & \multicolumn{3}{|l|}{456.68} & \multicolumn{6}{|c|}{464} \\
\hline Total number of plies ${ }^{5}$ & \multicolumn{3}{|l|}{465.63} & \multicolumn{6}{|l|}{464} \\
\hline
\end{tabular}

Table 11 Stacking sequence and lamination parameters of the panel at local level with 36 variables

\begin{tabular}{lrrrl}
\hline Panel no. & $V_{1}$ & $V_{2}$ & $V_{3}$ & Buckling load factor \\
\hline 16 & 1.1582 & 1.0070 & 1.2177 \\
17 & 1.1801 & 1.0081 & 1.2030 & \\
18 & 1.2398 & 1.0139 & 1.0987 & $1.0337\left(2^{\text {nd }}\right.$ buckling factor $)$ \\
7 & 1.2630 & 1.0547 & 0.8958 & \\
8 & 1.2604 & 1.0547 & 0.8958 & \\
9 & 1.2084 & 1.0296 & 1.1270 & $0.9614\left(1^{\text {st }}\right.$ buckling factor $)$ \\
Stacking sequence: & & \\
16 & {$\left[( \pm 45)_{2} /\left(0_{2} / 45 / 0_{2} /-45\right)_{2} / 0_{2} / 45 / 90 /-45 /(0 / 90)_{2} / 0_{2} / 90_{3} / 0 / 90 / 0_{3} / 90_{3} / 0 / 90 / 45 / 90 /-45 / 0 /(0 / 90)_{2} / 90_{2} /\right.$} \\
17 & $\left.(90 / 0)_{3} / 45 / 0 /-45 /( \pm 45)_{6} / 90_{2} / 45 / 90 /-45 /(45 / 0 /-45)_{2}\right]_{\mathrm{s}}$ \\
18 & {$\left[( \pm 45)_{2} /\left(0_{2} / 45 / 0_{2} /-45\right)_{2} / 0_{2} / 45 / 90 /-45 /(0 / 90)_{2} / 0_{2} / 90_{3} / 0 / 90 / 0_{3} / 90_{3} / 0 / 90 / 45 / 90 /-45 / 0 /(0 / 90)_{2} / 90_{2} /\right.$} \\
7 & $\left.(90 / 0)_{3} / 45 / 0 /-45 /( \pm 45)_{6}\right]_{\mathrm{s}}$ \\
8 & {$\left[( \pm 45)_{2} /\left(0_{2} / 45 / 0_{2} /-45\right)_{2} / 0_{2} / 45 / 90 /-45 /(0 / 90)_{2} / 0_{2} / 90_{3} / 0 / 90 / 0_{3} / 90_{3} / 0 / 90 / 45 / 90 /-45 / 0 /(0 / 90)_{2}\right]_{\mathrm{s}}$} \\
9 & {$\left[ \pm 45 / 0_{4} / 90 / 0\right]_{\mathrm{s}}$} \\
\end{tabular}

\section{Problem with Nine Designable Substructures}

Due to the limitation on the number of design variables in ANSYS, all panels in the top skin only are considered designable in the top level optimization and the configuration of panels in the bottom skin is fixed and is the same as the discrete optimal results for the case of six designable substructures above. In Table 12, it can be seen that the buckling is prevented in the discrete optimal design and the objective function was increased from 1171.9 to 1192 . Also, due to the blending requirements in the local optimization, the first buckling mode happens in bottom skin and the second in the top skin. These can be repaired by a manual adjustment. The results in Table 14 and 15 are obtained by the optimization with lamination parameter-based method. With the objective function to target the lamination parameters from the top level, the plies are shuffled with blending consideration. The buckling load factor has 
decreased $1 \%$ from 1.0213 at the top level to 1.015 at the local level. That shows that the lamination parameter-based method works well for the optimization of laminated composite structures if a small difference between the lamination parameters from top level optimization and the ones calculated in the local level can be produced. This can typically be achieved for realistic aircraft structures where the number of plies is not too small so that blending does not prevent from arriving at a good match of lamination parameters.

Table 12. Continuous and rounded optimal design with 27 variables for smeared stiffness-based method

\begin{tabular}{|c|c|c|c|c|c|c|c|}
\hline Panel no. & $\begin{array}{l}n_{0} \\
\text { (Con }\end{array}$ & $\begin{array}{c}n_{45} \\
\text { nuous) }\end{array}$ & $n_{90}$ & $\begin{array}{l}n_{0} \\
\text { (Ro }\end{array}$ & $\begin{array}{c}n_{45} \\
\text { aded) }\end{array}$ & $n_{90}$ & Active constraints \\
\hline 10 & 26.21 & 15.25 & 15.61 & 26 & 16 & 16 & \\
\hline 11 & 21.90 & 15.49 & 13.46 & 22 & 16 & 13 & \\
\hline 12 & 21.21 & 6.79 & 9.22 & 21 & 7 & 9 & buckling \\
\hline 13 & 25.40 & 7.12 & 6.86 & 25 & 8 & 7 & buckling \\
\hline 14 & 30.71 & 16.24 & 13.91 & 31 & 17 & 14 & \\
\hline 15 & 34.46 & 18.38 & 17.70 & 34 & 19 & 18 & \\
\hline 16 & 28.57 & 15.48 & 16.46 & 29 & 16 & 16 & \\
\hline 17 & 29.83 & 11.65 & 15.11 & 30 & 12 & 15 & \\
\hline 18 & 24.95 & 5.45 & 10.72 & 25 & 6 & 11 & \\
\hline Buckling load factor & \multicolumn{3}{|c|}{0.9967} & \multicolumn{3}{|c|}{1.0032} & \\
\hline Total number of plies & \multicolumn{3}{|c|}{1171.9} & \multicolumn{3}{|c|}{1192} & \\
\hline
\end{tabular}

Table 13 Stacking sequence of top skin panels for the rounded design with 27 variables

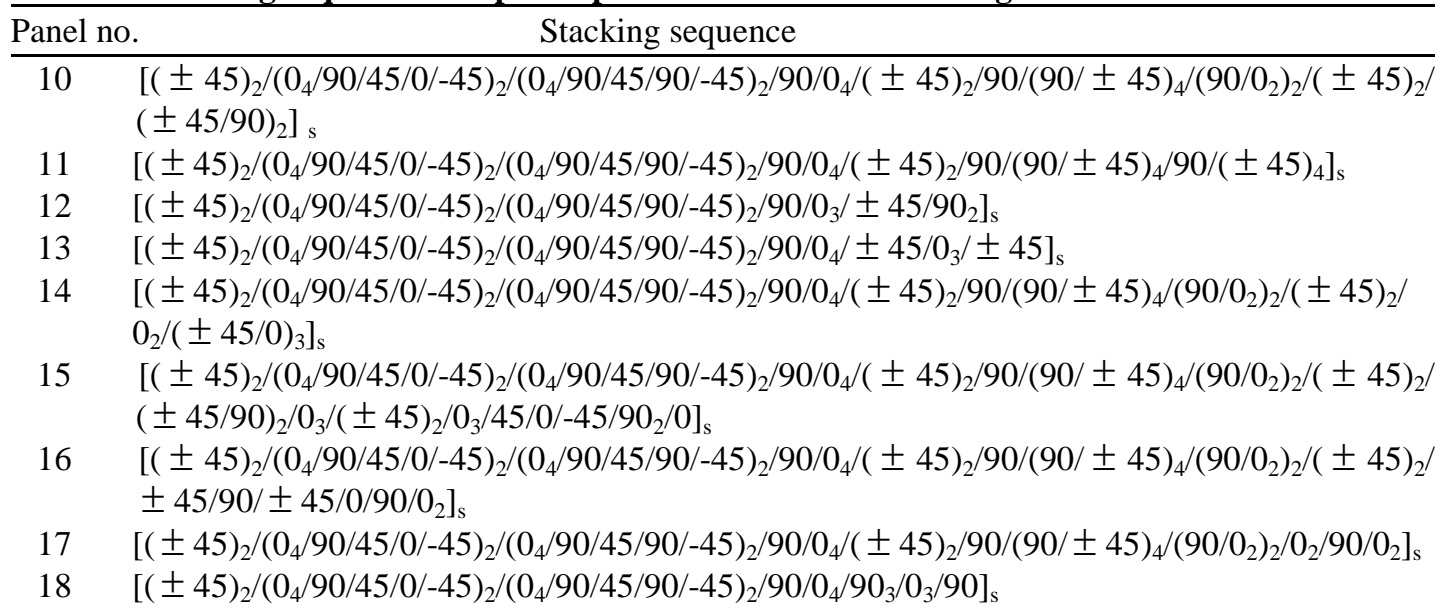

Buckling load factor $\quad 0.990\left(1^{\text {st }}\right.$ buckling of panel 9) $0.994\left(2^{\text {nd }}\right.$ buckling of panel 16$)$

Table 14 Continuous and rounded optimal design with 54 variables for lamination parameter-based method

\begin{tabular}{|c|c|c|c|c|c|c|c|c|c|}
\hline Panel no. & $\begin{array}{l}n_{0} \\
\text { (Con }\end{array}$ & $\begin{array}{c}n_{45} \\
\text { huous) }\end{array}$ & $n_{90}$ & $\begin{array}{l}n_{0} \\
(\mathrm{Rc}\end{array}$ & $\begin{array}{l}n_{45} \\
\text { nded }\end{array}$ & $n_{90}$ & $V_{1}$ & $V_{2}$ & $V_{3}$ \\
\hline 10 & 27.07 & 14.44 & 21.40 & 27 & 15 & 21 & 1.0978 & 1.0094 & 1.2446 \\
\hline 11 & 25.34 & 12.85 & 19.08 & 25 & 13 & 19 & 1.1261 & 1.0086 & 1.2905 \\
\hline 12 & 20.73 & 5.67 & 12.84 & 21 & 6 & 13 & 1.2319 & 1.0089 & 1.0736 \\
\hline 13 & 20.70 & 5.66 & 12.84 & 21 & 6 & 13 & 1.2311 & 1.0087 & 1.0745 \\
\hline 14 & 25.35 & 13.24 & 19.28 & 25 & 14 & 19 & 1.1189 & 1.0083 & 1.2596 \\
\hline 15 & 27.66 & 15.70 & 22.04 & 28 & 16 & 22 & 1.0947 & 1.0096 & 1.2001 \\
\hline 16 & 27.48 & 15.81 & 22.07 & 27 & 16 & 22 & 1.0987 & 1.0102 & 1.2013 \\
\hline 17 & 25.56 & 13.49 & 19.36 & 26 & 14 & 19 & 1.1224 & 1.0082 & 1.2492 \\
\hline 18 & 20.99 & 6.05 & 13.05 & 21 & 7 & 13 & 1.2243 & 1.0071 & 1.0460 \\
\hline Buckling load factor & \multicolumn{3}{|c|}{1.0014} & \multicolumn{3}{|c|}{1.0213} & & & \\
\hline Total number of plies & \multicolumn{3}{|c|}{1177.32} & \multicolumn{3}{|c|}{1192} & & & \\
\hline
\end{tabular}


Table 15 Stacking sequence and lamination parameters of the panel at local level with 54 variables

\begin{tabular}{ccccc}
\hline Panel no. & $V_{1}$ & $V_{2}$ & $V_{3}$ & Buckling load factor \\
\hline 10 & 1.1527 & 1.0080 & 1.1901 & \\
11 & 1.1685 & 1.0089 & 1.1774 & \\
12 & 1.2319 & 1.0143 & 1.0736 & \\
13 & 1.2319 & 1.0143 & 1.0736 & \\
14 & 1.1643 & 1.0086 & 1.1816 & \\
15 & 1.1457 & 1.0076 & 1.1934 & \\
16 & 1.1474 & 1.0077 & 1.1927 & \\
17 & 1.1623 & 1.0086 & 1.1833 & \\
18 & 1.2266 & 1.0135 & 1.0856 &
\end{tabular}

Stacking sequence:

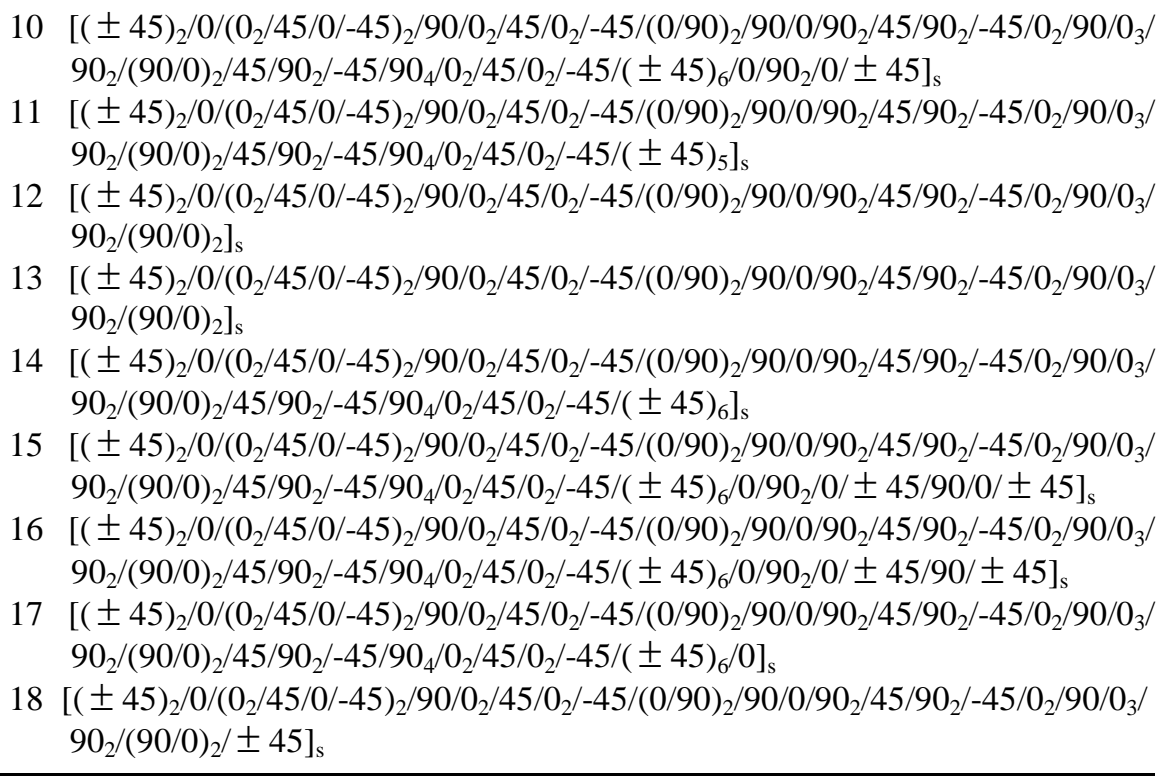

\section{Conclusions}

A bi-level composite optimization procedure was investigated and two approaches were examined for seeking the best stacking sequence of laminated composite wing structures with blending and manufacturing constraints.

In the smeared stiffness-based method, the top level optimization is performed using the assumption of homogenous laminates. A ply shuffling technique HyperShuffle is used at the local level without a need for solving an optimization problem. Manufacturing and general composite layup requirements are considered in the ply shuffling procedure. The obtained values of the buckling load factors for the case of two and six substructures do not violate the buckling constraints. For the case of nine substructures, a manual adjustments (adding layers) to the bottom skin layup following the blending and manufacturing requirements can prevent buckling.

In the lamination parameter-based method, local optimization runs need to be performed to shuffle layers while matching the lamination parameter values passed from the top level. The stacking sequence optimization in this level can also be done efficiently using a permutation GA because it does not call any numerical simulation and only deals with calculating the lamination parameter values by simple formulae. Once the stacking sequence is determined that satisfies the blending and manufacturing requirements, no buckling analysis needs to be performed if the target values of the lamination parameters, passed from the top level, were kept. Based on three cases in this paper, it seems difficult to match the lamination parameters from the top level while considering ply continuity for a small number of plies in the bottom skin of the laminated composite wing box. Such problems did not occur for a more realistic case of a larger number of plies, as in the top skin.

\section{References}

${ }^{1}$ Gurdal, Z., Haftka, R.T., and Hajela, P., Design and Optimization of Laminated Composite Materials, John Wiley \& Sons, 1999.

${ }^{2}$ Schmit, L.A., and Farshi, B., "Optimum Laminate Design for Strength and Stiffness," International Journal for Numerical Methods in Engineering, Vol. 7, 1973, pp. 519-536. 
${ }^{3}$ Stroud, W.J., and Agranoff, N., "Minimum Mass Design of Filamentary Composite Panels under Combined Loads: Design Procedure Based on Simplified Equations," NASA TN D-8257, 1976.

${ }^{4}$ Nemeth, M.P., "Importance of Anisotropy on Buckling of Compression-Loaded Symmetric Composite Plates," AIAA Journal Vol. 24, 1986, pp. 1831-1835.

${ }^{5}$ Liu, B., Haftka, R.T., and Akgun, M.A., "Two-Level Composite Wing Structural Optimization Using Response Surface," Structural and Multidisciplinary Optimization, Vol. 20, 2000, pp. 87-96.

${ }^{6}$ Liu, B., Haftka, R.T., and Trompette, P., "Maximisation of Buckling Loads of Composite Panels Using Flexural Lamination Parameters," Structural and Multidisciplinary Optimization, Vol. 26, 2004, pp. 28-36.

${ }^{7}$ Tsai, S.W., Halpin, J.C., and Pagano, N.J., "Composite Materials Workshop," Technomic publishing Co., Westport, Connecticut, 1968 , pp. 233-253.

${ }^{8}$ Fukunaga, H., and Hirano, Y., "Stability Optimization of Laminated Composite Plates under In-Plane Loads," Proceedings of the fourth International Conference on Composite Materials, Japan, 1982, pp 565-572.

${ }^{9}$ Miki, M., "Material Design of Composite Laminates with Required In-Plane Elastic Properties," Proceedings of the fourth International Conference on Composite Materials, Japan, 1982, pp 1725-1731.

${ }^{10}$ Fukunaga, H., and Chou, T.W., "On Laminated Configurations for Simultaneous Failure," Journal of Composite Materials, Vol. 22, 1988, pp. 271-286.

${ }^{11}$ Diaconu, C.G., Sato, M., and Sekine, H., "Feasible Region in General Design Space of Lamination Parameters for Laminated Composites," AIAA Journal, Vol. 40, 2002, pp. 559-565.

${ }^{12}$ Herencia, J.E., and Weaver, P.M., "Local Optimization of Long Anisotropic Laminated Fibre Composite Panels with T Shape Stiffeners," Proceedings of 47th AIAA SDM Conference, AIAA 2006-2171, Newport, Rhode Island, May. 2006.

${ }^{13}$ Herencia, J.E., Haftka, R.T., Weaver, P.M., and Friswell, M.I., "Optimization of Anisotropic Composite Panels with TShaped Stiffeners Using Linear Approximations of the Design Constraints to Identify Their Stacking Sequence," Proceedings of 7th ASMO UK/ISSMO Conference, Bath, UK, 2008.

${ }^{14}$ Liu, B., and Haftka, R.T., "Composite Wing Structural Design Optimization with Continuity Constraint," Proceedings of $42^{\text {th }}$ AIAA SDM Conference, AIAA-2001-1205, Seattle, WA, 2001

${ }^{15}$ Soremekun, G.A., Gurdal, Z., Kassapoglou, C., and Toni, D., "Stacking Sequence Blending of Multiple Composite Laminates Using Genetic Algorithm," Composite Structures, Vol. 56, 2002, pp. 53-62.

${ }^{16}$ Seresta, O., Gurdal, Z., Adams, D.B., and Watson, L.T., "Optimal Design of Composite Wing Structures with Blended Laminates," Composites Part B: Engineering, Vol. 38, 2007, pp. 469-480.

${ }^{17}$ Toropov, V.V., Jones, R., Willment, T., and Funnell, M., "Weight and Manufacturability Optimization of Composite Aircraft Components Based on A Genetic Algorithm," Proceedings of $6^{\text {th }}$ World Congress of SMO, Brazil, 2005.

${ }^{18}$ Liu, W., and Krog, L., "A Method for Composite Ply Layout Design and Stacking Sequence Optimisation," Proceedings of 7th ASMO UK/ISSMO Conference, Bath, UK, 2008.

${ }^{19}$ Jones, R.M., Mechanics of Composite Materials, Second Edition, Taylor and Francis, 1999.

${ }^{20}$ Zhou, M., and Fleury, R., "Optimization of Composite Structures-Understanding and Meeting the Challenges," Altair Composite Seminar, Royal Lemington Spa, UK, 2007.

${ }^{21}$ Altair OptiStruct Version 9.0, Altair Engineering Inc., 2008.

${ }^{22}$ Ansys Elements Reference, Ver.11, ANSYS, 2007.

${ }^{23}$ Schramm, U., “Altair's Product Direction Solver Technologies," European HyperWorks Technology Conference 2007, Berlin, Germany, October, 2007.

${ }^{24}$ Niu, M.C.Y., Composite Airframe Structures, Practical Design Information and Data, Conmilit Press Ltd., Hong Kong. 1992. 